\title{
Degree-regular triangulations of the double-torus
}

\author{
Basudeb Datta and Ashish Kumar Upadhyay
}

To appear in 'Forum Mathematicum'

\begin{abstract}
A connected combinatorial 2-manifold is called degree-regular if each of its vertices have the same degree. A connected combinatorial 2-manifold is called weakly regular if it has a vertex-transitive automorphism group. Clearly, a weakly regular combinatorial 2-manifold is degree-regular and a degree-regular combinatorial 2-manifold of Euler characteristic -2 must contain 12 vertices.

In 1982, McMullen et al. constructed a 12-vertex geometrically realized triangulation of the double-torus in $\mathbb{R}^{3}$. As an abstract simplicial complex, this triangulation is a weakly regular combinatorial 2-manifold. In 1999, Lutz showed that there are exactly three weakly regular orientable combinatorial 2-manifolds of Euler characteristic -2 . In this article, we classify all the orientable degree-regular combinatorial 2-manifolds of Euler characteristic -2 . There are exactly six such combinatorial 2-manifolds. This classifies all the orientable equivelar polyhedral maps of Euler characteristic -2 .
\end{abstract}

AMS classification : 57Q15, 57M20, 57N05.

Keywords : Triangulations of 2-manifolds, regular simplicial maps, combinatorially regular triangulations, degree-regular triangulations.

\section{Introduction}

Recall that an (abstract) simplicial complex is a collection of nonempty finite sets (set of vertices) such that every nonempty subset of a member is also a member. For $i \geq 0$, the elements of size $i+1$ are called the $i$-simplices of the simplicial complex. 1-simplices are also called the edges of the simplicial complex. For a simplicial complex $X$, the maximum of $k$ such that $X$ has a $k$-simplex is called the dimension of $X$. The set $V(X)$ of vertices of $X$ is called the vertex-set of $X$. A simplicial complex $X$ is called finite if $V(X)$ is finite.

If $X$ and $Y$ are two simplicial complexes, then a (simplicial) isomorphism from $X$ to $Y$ is a bijection $\varphi: V(X) \rightarrow V(Y)$ such that for $\sigma \subseteq V(X), \sigma$ is a simplex of $X$ if and only if $\varphi(\sigma)$ is a simplex of $Y$. Two simplicial complexes $X, Y$ are called isomorphic (denoted by $X \cong Y$ ) when such an isomorphism exists. We identify two simplicial complexes if they are isomorphic. An isomorphism from a simplicial complex $X$ to itself is called an automorphism of $X$. All the automorphisms of $X$ form a group, which is denoted by $\operatorname{Aut}(X)$.

A simplicial complex $X$ is usually thought of as a prescription for constructing a topological space (called the geometric carrier of $X$ and is denoted by $|X|$ ) by pasting together geometric simplices. Formally, $|X|$ is the subspace of $[0,1]^{V(X)}$ consisting of the functions $f: V(X) \rightarrow[0,1]$ such that the support $\{v \in V(X): f(v) \neq 0\}$ is a simplex of $X$ and $\sum_{v \in V(X)} f(v)=1$. If $\sigma$ is a simplex then $|\sigma|:=\left\{f \in|X|: \sum_{v \in \sigma} f(v)=1\right\}$ is called the geometric simplex corresponding to $\sigma$. We say that a simplicial complex $X$ triangulates a topological space $Y$ if $Y$ is homeomorphic to $|X|$. A simplicial complex $X$ is called connected 
if $|X|$ is connected. A 2-dimensional simplicial complex is called a combinatorial 2-manifold if it triangulates a closed surface. A combinatorial 2-manifold $X$ is called orientable if $|X|$ is an orientable 2 -manifold.

For a simplicial complex $X$, an embedding $i:|X| \hookrightarrow \mathbb{R}^{n}$ is called geometric if the image of the geometric simplices are convex. A triangulation $K$ of a surface is called geometrically realizable in $\mathbb{R}^{3}$ if $|K|$ can be embedded geometrically in $\mathbb{R}^{3}$.

If $v$ is a vertex of a simplicial complex $X$, then the number of edges containing $v$ is called the degree of $v$ and is denoted by $\operatorname{deg}_{X}(v)(\operatorname{or} \operatorname{deg}(v))$. A simplicial complex is called neighbourly if each pair of vertices form an edge.

A pattern is an ordered pair $(M, G)$, where $M$ is a connected closed surface and $G$ is a finite graph on $M$ such that each component of $M \backslash G$ is simply connected. The closure of each component of $M \backslash G$ is called a face of $(M, G)$. A pattern is called a polyhedral pattern if the boundary of each face is a cycle and the intersection of any two faces is empty, a vertex or an edge (cf. [10]). A polyhedral map is the collection of the boundary cycles (together with their vertices and edges) of a polyhedral pattern (cf. [4]). So, a combinatorial 2-manifold is a polyhedral map. For a polyhedral map $K$, consider the polyhedral map $\widetilde{K}$ (called the dual of $K)$ whose vertices are the faces of $K$ and $\left(F_{1}, \ldots, F_{m}\right)$ is a face of $\widetilde{K}$ if $F_{1}, \ldots, F_{m}$ have a common vertex and $F_{1} \cap F_{2}, \ldots, F_{m-1} \cap F_{m}, F_{m} \cap F_{1}$ are edges. For a combinatorial definition of polyhedral maps see [14].

A polyhedral map is called $\{p, q\}$-equivelar if each face is a $p$-cycle and each vertex is in $q$ faces. A polyhedral map is called equivelar if it is $\{p, q\}$-equivelar for some $p, q$ (cf. $[14,15])$. Clearly, a polyhedral map $K$ is $\{p, q\}$-equivelar if and only if $\widetilde{K}$ is $\{q, p\}$-equivelar.

Since the degrees of the vertices in an equivelar combinatorial 2-manifold are same, an equivelar combinatorial 2-manifold is also called degree regular. More explicitly, a connected combinatorial 2-manifold $X$ is said to be degree-regular of type $d$ if each vertex of $X$ has degree $d$. A combinatorial 2-manifold is said to be degree-regular if it is degree-regular of type $d$ for some $d$. So, trivial examples of degree-regular combinatorial 2-manifolds are neighbourly combinatorial 2-manifolds.

A combinatorially regular combinatorial 2-manifold is a connected combinatorial 2manifold with a flag-transitive automorphism group (a flag is a triple $(u, e, F$ ), where $e$ is an edge of the face $F$ and $u$ is a vertex of $e$ ). A connected combinatorial 2-manifold $X$ is said to be weakly regular if the automorphism group of $X$ acts transitively on $V(X)$. Clearly, a combinatorially regular combinatorial 2-manifold is weakly regular and a weakly regular combinatorial 2-manifold is degree-regular. If a degree-regular (respectively, weakly regular) combinatorial 2-manifold $X$ triangulates a space $Y$ then we say $X$ is a degree-regular (respectively, weakly regular) triangulation of $Y$.

If the number of $i$-simplices of a $d$-dimensional finite simplicial complex $X$ is $f_{i}(X)$ $(0 \leq i \leq d)$, then the number $\chi(X):=\sum_{i=0}^{d}(-1)^{i} f_{i}(X)$ is called the Euler characteristic of $X$. Similarly, for a polyhedral map $K$, the number of vertices - the number of edges + the number of faces is called the Euler characteristic of $K$.

For the existence of an $n$-vertex neighbourly combinatorial 2-manifold, $n(n-1)$ must be divisible by 6 , equivalently, $n \equiv 0$ or $1 \bmod 3$. Ringel and Jungerman $([11,16])$ have shown that there exist neighbourly combinatorial 2-manifolds on $3 k$ and $3 k+1$ vertices, for each $k \geq 2$. In [2], Altshuler and Brehm have shown that there are exactly 19 neighbourly combinatorial 2-manifolds on at most 11 vertices. By using computer, Altshuler et al ([1]) have shown that there are exactly 59 orientable neighbourly combinatorial 2-manifolds on 12 vertices. In [3], Bokowski and Guedes de Oliveira have shown that one of these 59 combinatorial 2-manifolds (namely, $N_{54}^{12}$ ) is not geometrically realizable in $\mathbb{R}^{3}$. In [8], Datta and 
Nilakantan have shown that there are exactly 27 degree-regular combinatorial 2-manifolds on at most 11 vertices.

If the degree of each vertex in an $n$-vertex combinatorial 2-manifold $K$ is $d$ then $n d=$ $2 f_{1}(K)=3 f_{2}(K)$ and $\chi(K)=f_{0}(K)-f_{1}(K)+f_{2}(K)=n-\frac{n d}{2}+\frac{n d}{3}=\frac{n(6-d)}{6}$. So, if $K$ is weakly regular and $\chi(K) \geq 0$ then $(n, d)=(4,3),(6,4),(6,5),(12,5)$ or $(\chi(K), d)=$ $(0,6)$. For each $(n, d) \in\{(4,3),(6,4),(6,5),(12,5)\}$, there exists a unique combinatorial 2manifold, namely, the 4 -vertex 2 -sphere, the boundary of the octahedron, the 6 -vertex real projective plane and the boundary of the icosahedron (cf. $[5,8]$ ). These 4 combinatorial 2-manifolds are combinatorially regular. Any degree-regular triangulation of the torus is weakly regular and for each $n \geq 7$, there exists an $n$-vertex weakly regular triangulation of the torus. There exists an $n$-vertex degree-regular triangulation of the Klein bottle if and only if $n$ is a composite number $\geq 9$ and for $k \geq 2$, there exists a $(4 k+2)$-vertex weakly regular triangulation of the Klein bottle (cf. [8,9]). There are infinitely many combinatorially regular triangulations of the torus whereas there is no such triangulation of the Klein bottle (cf. [19]). For the existence of degree-regular of type $d$ combinatorial 2 -manifolds of negative Euler characteristic, $d$ must be at least 7 . Since $\frac{n(6-d)}{6} \neq-1$ for $n>d \geq 7$, there does not exist any degree-regular combinatorial 2-manifolds of Euler characteristic -1 . Schulte and Wills $([17,18])$ have constructed two combinatorially regular geometrically realized triangulations of the orientable surface of genus 3 in $\mathbb{R}^{3}$.

The orientable surface of genus 2 (the double-torus) has Euler characteristic -2 . It is known that for a triangulation of the double-torus one needs at least 10 vertices. In [13], Lutz has shown that there are exactly 865 distinct 10 -vertex triangulations of the double torus and all of them are geometrically realizable in $\mathbb{R}^{3}$. If $K$ is an $n$-vertex degreeregular of type $d$ combinatorial 2-manifold of Euler characteristic -2 then $-2=\frac{n(6-d)}{6}$ and hence $(n, d)=(12,7)$. In [15], McMullen et al. constructed a weakly regular triangulation (isomorphic to $N_{1}$ of Example 1) of the double-torus which has a linear geometric realization in $\mathbb{R}^{3}$. In [12], Lutz showed that there are exactly 3 orientable weakly regular combinatorial 2-manifolds of Euler characteristic -2 (i.e., triangulations of the double-torus). These 3 are $N_{1}, N_{2}$ and $N_{3}$ of Example 1. Lutz also showed that $\operatorname{Aut}\left(N_{1}\right) \cong \operatorname{Aut}\left(N_{2}\right) \cong \mathbb{Z}_{12}$ and $\operatorname{Aut}\left(N_{3}\right) \cong D_{6}$. Here we prove the following:

Theorem 1. Let $N_{1}, \ldots, N_{6}$ be as in Example 1. Then

(a) $N_{i} \neq N_{j}$ for $1 \leq i<j \leq 6$,

(b) $\operatorname{Aut}\left(N_{4}\right) \cong \mathbb{Z}_{2}, \operatorname{Aut}\left(N_{5}\right) \cong D_{3}, \operatorname{Aut}\left(N_{6}\right) \cong \mathbb{Z}_{2} \times \mathbb{Z}_{2}$, and

(c) if $M$ is an orientable degree-regular combinatorial 2-manifold of Euler characteristic -2 then $M$ is isomorphic to one of $N_{1}, \ldots, N_{6}$.

If there exists an $n$-vertex $\{p, q\}$-equivelar polyhedral map of Euler characteristic -2 then $-2=n-\frac{n q}{2}+\frac{n q}{p}$ and $\frac{n q(p-3)}{2}+\frac{n q}{2} \leq\left(\begin{array}{c}n \\ 2\end{array}\right)$. So, $(n, p, q)=(12,3,7)$ or $(28,7,3)$. Since two polyhedral maps $M$ and $N$ are isomorphic if and only if their duals $\widetilde{M}$ and $\widetilde{N}$ are isomorphic (cf. [14]), from Theorem 1, we get.

COROLlary 2. There are exactly 12 orientable equivelar polyhedral maps of Euler characteristic -2 , namely, $N_{1}, \ldots, N_{6}, \widetilde{N_{1}}, \ldots, \widetilde{N_{5}}$ and $\widetilde{N_{6}}$. 


\section{Examples and Preliminaries}

In this section we present those degree-regular combinatorial 2-manifolds and their automorphism groups mentioned in the previous section. First we give some definitions and notations which will be used throughout the paper.

A 2-simplex in a 2-dimensional simplicial complex is also said to be a face. We denote a face $\{u, v, w\}$ by $u v w$. We also denote an edge $\{u, v\}$ by $u v$.

A graph is an one dimensional simplicial complex. The complete graph on $n$ vertices is denoted by $K_{n}$. Disjoint union of $m$ copies of $K_{n}$ is denoted by $m K_{n}$. A graph without any edge is called a null graph. An $n$-vertex null graph is denoted by $\emptyset_{n}$.

If $G$ is a graph and $n \geq 0$ is an integer then we consider the graph $G_{n}(G)$ as follows. The vertices of $G_{n}(G)$ are the vertices of $G$. Two vertices $u$ and $v$ form an edge in $G_{n}(G)$ if the number of common neighbours of $u$ and $v$ is $n$. Clearly, if $G$ and $H$ are isomorphic then $G_{n}(G)$ and $G_{n}(H)$ are isomorphic for all $n \geq 0$.

A connected finite graph is called a cycle if the degree of each vertex is 2 . An $n$ cycle is a cycle on $n$ vertices and is denoted by $C_{n}$ (or by $C_{n}\left(a_{1}, \ldots, a_{n}\right.$ ) if the edges are $\left.a_{1} a_{2}, \ldots, a_{n-1} a_{n}, a_{n} a_{1}\right)$. Disjoint union of $m$ copies of $C_{n}$ is denoted by $m C_{n}$.

For a simplicial complex $K$, the graph consisting of the edges and vertices of $K$ is called the edge-graph of $K$ and is denoted by $\operatorname{EG}(K)$. Let $K$ be a simplicial complex with vertexset $V(K)$. If $U \subseteq V(K)$ then the induced subcomplex of $K$ on $U$ (denoted by $K[U]$ ) is the subcomplex whose simplices are those of $K$ which are subsets of $U$.

If $v$ is a vertex of a simplicial complex $X$, then the link of $v$ in $X$, denoted by $\operatorname{lk}_{X}(v)$ (or $\mathrm{lk}(v)$ ), is the simplicial complex $\{\tau \in X: v \notin \tau,\{v\} \cup \tau \in X\}$. If $v$ is a vertex of a simplicial complex $X$, then the star of $v$ in $X$, denoted by st $X(v)$ (or st $(v)$ ), is the simplicial complex $\left\{\{v\}, \tau, \tau \cup\{v\}: \tau \in \mathrm{lk}_{X}(v)\right\}$. Clearly, a finite simplicial complex $K$ is a combinatorial 2-manifold if and only if $\operatorname{lk}_{K}(v)$ is a cycle for each vertex $v$ of $K$.

EXAmPLE 1. Six degree-regular combinatorial 2-manifolds of Euler characteristic -2. Each of them is orientable and hence triangulates the connected sum of two copies of the torus (the double-torus). The vertex-set of each of these is $\{0,1, \ldots, 11\}$.
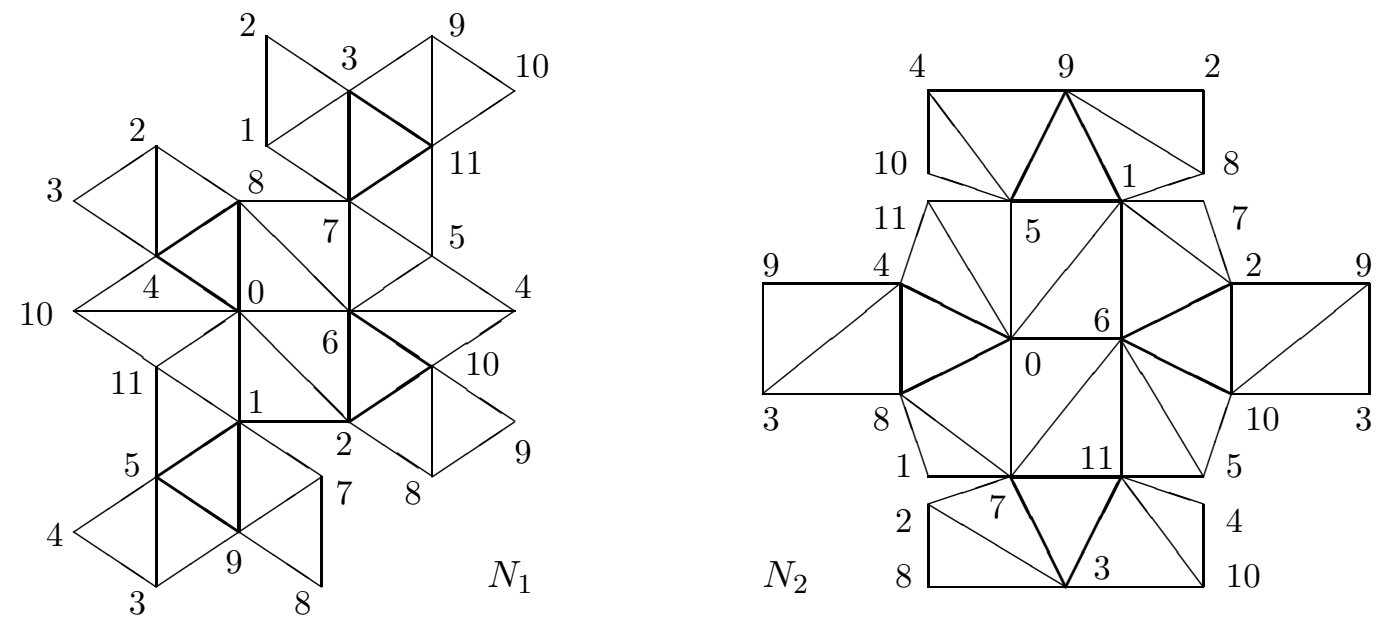

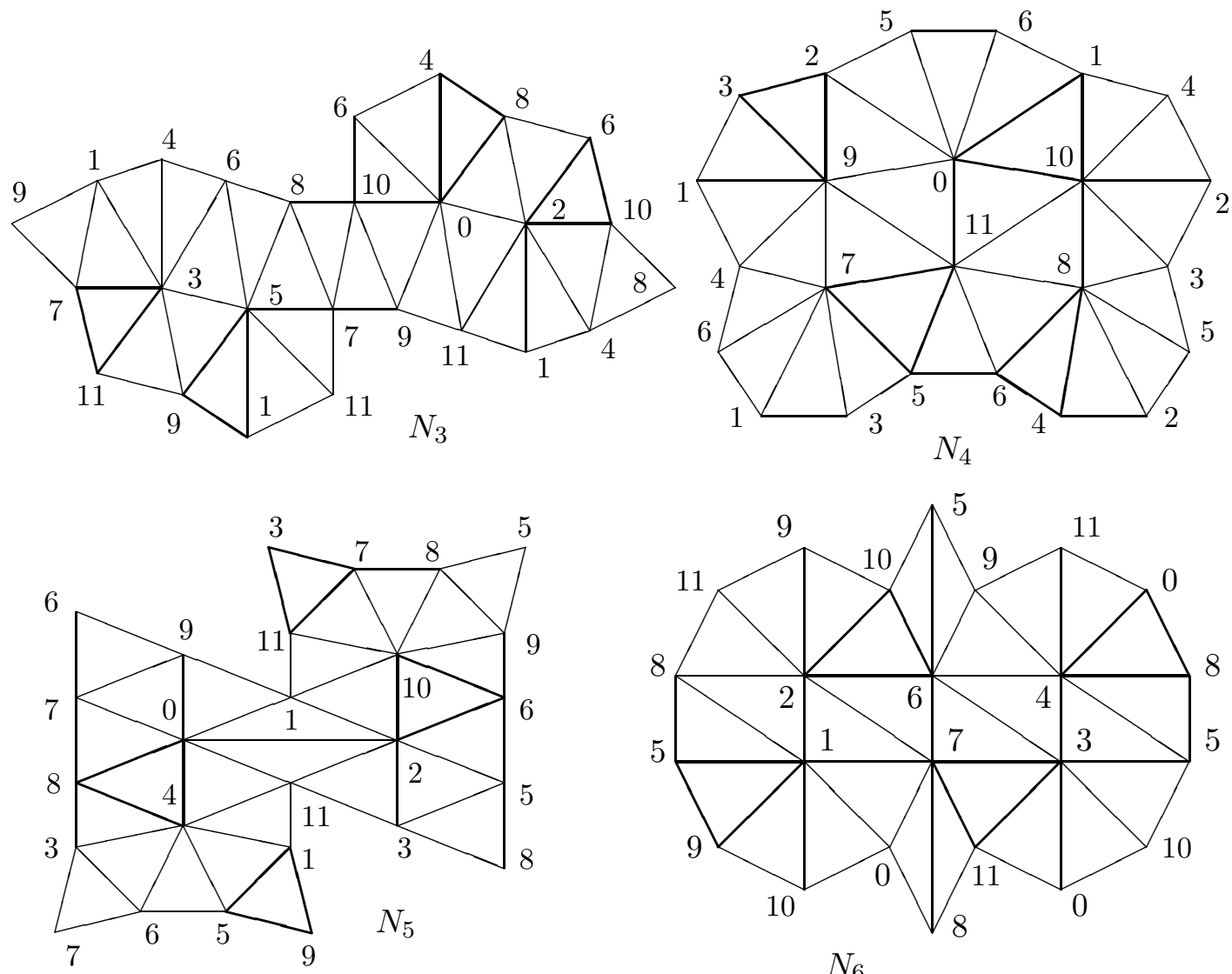

Lemma 2.1. If $N_{1}, \ldots, N_{6}$ are as in Example 1 then $N_{i} ¥ N_{j}$ for $1 \leq i<j \leq 6$.

Proof. If $N_{i} \cong N_{j}$ then $\operatorname{EG}\left(N_{i}\right) \cong \operatorname{EG}\left(N_{j}\right)$ and hence $G_{n}\left(\operatorname{EG}\left(N_{i}\right)\right) \cong G_{n}\left(\operatorname{EG}\left(N_{j}\right)\right.$ ) (as graphs) for all $n \geq 0$.

Observe that $G_{2}\left(\operatorname{EG}\left(N_{1}\right)\right)=C_{12}(0,1, \ldots, 11), G_{2}\left(\operatorname{EG}\left(N_{2}\right)\right)=\emptyset_{12}, G_{2}\left(\operatorname{EG}\left(N_{3}\right)\right)=$ $C_{12}(0,11,2,1,4,3,6,5,8,7,10,9), G_{2}\left(\mathrm{EG}\left(N_{4}\right)\right)=(\{0,1, \ldots, 11\},\{\{9,11\},\{10,11\}\})$, $G_{2}\left(\operatorname{EG}\left(N_{5}\right)\right)=(\{0,1, \ldots, 11\},\{\{0,2\},\{4,6\},\{8,10\}\})$ and $G_{2}\left(\operatorname{EG}\left(N_{6}\right)\right)=(\{0,1, \ldots, 11\}$, $\{\{5,8\},\{6,7\},\{9,11\},\{10,0\}\})$. So, we need to show only that $N_{1} \neq N_{3}$.

Observe that $G_{5}\left(\operatorname{EG}\left(N_{1}\right)\right)=G_{5}\left(\operatorname{EG}\left(N_{3}\right)\right)=C_{6}(1,3,5,7,9,11) \cup C_{6}(0,2,4,6,8,10)$. If $\varphi: N_{1} \rightarrow N_{3}$ is an isomorphism then we may assume that $\varphi(0)=0$ (since $\mathbb{Z}_{12}$ acts vertextransitively on $\left.N_{1}\right)$. Since $\varphi$ induces an isomorphism from $G_{5}\left(\operatorname{EG}\left(N_{1}\right)\right)$ to $G_{5}\left(\operatorname{EG}\left(N_{3}\right)\right)$, $\varphi(\{0,2, \ldots, 10\})=\{0,2, \ldots, 10\}$ and hence $\varphi(\{1,3, \ldots, 11\})=\{1,3, \ldots, 11\}$. Then, from $\mathrm{lk}_{N_{1}}(0)$ and $\mathrm{lk}_{N_{3}}(0), \varphi(\{1,11\})=\{9,11\}$. If $\varphi(1)=11$ then, from $\mathrm{lk}_{N_{1}}(0), \mathrm{lk}_{N_{1}}(1), \mathrm{lk}_{N_{3}}(0)$ and $\operatorname{lk}_{N_{3}}(11), \varphi=(11,9,7,5,3,1)(4,6,8)$. Now, $\{2,3,4\}$ is a face of $N_{1}$ but $\varphi(\{2,3,4\})=$ $\{1,2,6\}$ is not a face of $N_{3}$, a contradiction. If $\varphi(1)=9$ then from $\mathrm{lk}_{N_{1}}(0), \mathrm{lk}_{N_{1}}(1)$, $\mathrm{lk}_{N_{3}}(0)$ and $\mathrm{lk}_{N_{3}}(9)$, we get $\varphi=(1,9,5,3,7)(2,10)(4,8)$. Now, $\{5,7,11\}$ is a face of $N_{1}$ but $\varphi(\{5,7,11\})=\{1,3,11\}$ is not a face of $N_{3}$, a contradiction. This completes the proof.

In [12], Lutz showed that $\operatorname{Aut}\left(N_{1}\right)=\operatorname{Aut}\left(N_{2}\right)=\langle\sigma\rangle \cong \mathbb{Z}_{12}$ and $\operatorname{Aut}\left(N_{3}\right)=\left\langle\sigma^{2}, \tau\right\rangle \cong D_{6}$, where $\sigma=(0,1, \ldots, 11)$ and $\tau=(1,4)(2,3)(8,9)(6,11)(0,5)(7,10)$. Here we prove.

Lemma 2.2. $\operatorname{Aut}\left(N_{4}\right)=\langle\gamma\rangle \cong \mathbb{Z}_{2}, \operatorname{Aut}\left(N_{5}\right)=\langle\alpha, \beta\rangle \cong D_{3}$ and $\operatorname{Aut}\left(N_{6}\right)=\left\langle\alpha_{1}, \alpha_{2}\right\rangle \cong$ $\mathbb{Z}_{2} \times \mathbb{Z}_{2}$, where $\alpha=(0,4,8)(1,5,9)(2,6,10)(3,7,11), \beta=(0,2)(4,10)(1,11)(3,9)(5,7)(6,8)$, 
$\alpha_{1}=(1,2)(3,4)(5,6)(7,8)(9,10)(11,0), \alpha_{2}=(1,3)(2,4)(5,7)(6,8)(9,11)(10,0)$ and $\gamma=$ $(1,2)(3,4)(5,6)(7,8)(9,10)$.

Proof. For $i \geq 0$ and $14 \leq j \leq 6$, let $\mathcal{E}_{i, j}$ denote the edge-set of $G_{i}\left(\operatorname{EG}\left(N_{j}\right)\right)$. Then $\mathcal{E}_{2,4}=\left\{\{9,11\},\{11,10\}, \mathcal{E}_{6,4}=\{\{3,4\},\{9,10\}\}\right.$ and $\mathcal{E}_{5,4}=\{\{11,1\},\{11,2\},\{11,3\},\{11,4\}$, $\{0,3\},\{0,4\},\{0,7\},\{0,8\},\{1,2\},\{7,8\},\{5,9\},\{5,10\},\{6,9\},\{6,10\}\}$.

Clearly, $\gamma$ is an automorphism of $N_{4}$ of order 2. Let $f$ be an automorphism. Then $f$ induces an automorphism (also denoted by $f)$ on $G_{i}\left(\operatorname{EG}\left(N_{4}\right)\right.$ ) for all $i \geq 0$. Therefore, considering the action of $f$ on $\mathcal{E}_{2,4}, f(11)=11$ and hence, considering the action of $f$ on $\mathcal{E}_{5,4}, f(0)=0$. Then, considering the faces through the edge $\{0,11\}, f(9)=9$ or 10 . In the first case, $f(10)=10$. Then, from the links of 11 and 0 in $N_{4}, f=\operatorname{Id}_{N_{4}}$. In the second case, $(\gamma \circ f)(9)=9$ and hence, by the previous case, $\gamma \circ f=\operatorname{Id}_{N_{4}}$. Thus, $f=\gamma^{-1}=\gamma$. This implies that $\operatorname{Aut}\left(N_{4}\right)=\left\{\operatorname{Id}_{N_{4}}, \gamma\right\} \cong \mathbb{Z}_{2}$.

Clearly, $\alpha, \beta \in \operatorname{Aut}\left(N_{5}\right)$. Also, $\alpha^{3}=\beta^{2}=\operatorname{Id}_{N_{5}}, \alpha \circ \beta=\beta \circ \alpha^{2}$ and $\alpha^{2} \circ \beta=\beta \circ \alpha$. So, $\langle\alpha, \beta\rangle \cong D_{3}$.

Claim 1. The identity is the only automorphism of $N_{5}$ which fixes the vertex 0 .

Observe that $G_{5}\left(\operatorname{EG}\left(N_{5}\right)\right)=C_{4}(0,3,10,5) \cup C_{4}(4,7,2,9) \cup C_{4}(8,11,6,1), \mathcal{E}_{2,5}=\{\{0,2\}$, $\{4,6\},\{8,10\}\}$ and $\mathcal{E}_{6,5}=\{\{0,10\},\{2,4\},\{6,8\}\}$.

If $g$ is an automorphism of $N_{5}$ then $g$ is also an automorphism of $G_{i}\left(\operatorname{EG}\left(N_{5}\right)\right)$ for all $i \geq 0$. Now, if $g(0)=0$ then, from the action of $g$ on $\mathcal{E}_{2,5}, g(2)=2$. These imply, considering the action of $g$ on $\mathcal{E}_{6,5}$ and $\mathcal{E}_{2,5}, g(10)=10, g(4)=4, g(6)=6$ and $g(8)=8$. Then, from the links of 0 and 2 in $N_{5}, g=\operatorname{Id}_{N_{5}}$. This proves the claim.

Now, let $f \in \operatorname{Aut}\left(N_{5}\right)$. Then, from the action of $f$ on $\mathcal{E}_{2,5}, f(0)=0,2,4,6,8$ or 10 .

If $f(0)=0$ then, by the claim, $f=\operatorname{Id}_{N_{5}}$. If $f(0)=2$ then $(\beta \circ f)(0)=0$ and hence, by Claim $1, \beta \circ f=\operatorname{Id}_{N_{5}}$. This implies that $f=\beta^{-1}=\beta$. If $f(0)=6$ then $\left(\beta \circ \alpha^{2} \circ f\right)(0)=0$ and hence, by Claim 1, $\beta \circ \alpha^{2} \circ f=\operatorname{Id}_{N_{5}}$. This implies that $f=\left(\beta \circ \alpha^{2}\right)^{-1}=\alpha \circ \beta$. Similarly, $f(0)=4$ implies $f=\alpha, f(0)=8$ implies $f=\alpha^{2}$ and $f(0)=10$ implies $f=\alpha^{2} \circ \beta$. Thus, $f \in\langle\alpha, \beta\rangle$. This implies that $\operatorname{Aut}\left(N_{5}\right)=\langle\alpha, \beta\rangle \cong D_{3}$.

Clearly, $\alpha_{1}$ and $\alpha_{2}$ are order 2 automorphisms of $N_{6}$. Also, $\alpha_{1}^{2}=\alpha_{2}^{2}=\operatorname{Id}_{N_{6}}$ and $\alpha_{1} \circ \alpha_{2}=\alpha_{2} \circ \alpha_{1}$. Thus, $\left\langle\alpha_{1}, \alpha_{2}\right\rangle \cong \mathbb{Z}_{2} \times \mathbb{Z}_{2}$.

Claim 2. The identity is the only automorphism of $N_{6}$ which fixes the vertex 1 .

Observe that $\mathcal{E}_{2,6}=\{\{5,8\},\{6,7\},\{9,11\},\{0,10\}\}$ and $\mathcal{E}_{3,6}=\{\{1,0\},\{1,9\},\{1,5\}$, $\{1,7\},\{3,5\},\{3,7\},\{3,10\},\{3,11\},\{2,10\},\{2,11\},\{2,6\},\{2,8\},\{4,6\},\{4,8\},\{4,0\}$, $\{4,9\}\}$.

If $g$ is an automorphism of $N_{6}$ then $g$ is also an automorphism of $G_{i}\left(\operatorname{EG}\left(N_{6}\right)\right)$ for all $i \geq 0$. Therefore, from the action of $g$ on $\mathcal{E}_{3,6}, g(\{1,2,3,4\})=\{1,2,3,4\}$. Now, let $g(1)=1$. Since $\{1,2\}$ is an edge of $N_{6}$ and $\{1,3\},\{1,4\}$ are non-edges of $N_{6}, g(2)=2$ and hence $g(\{3,4\})=\{3,4\}$. Since 127, 128,345,346 are faces of $N_{6}, g(\{7,8\})=\{7,8\}$ and $g(\{5,6\})=\{5,6\}$. Then, from the link of $1, g(5)=5$ and hence $g(8)=8, g(7)=7$, $g(9)=9, g(10)=10, g(0)=0$ and $g(6)=6$. Now, from the link of $5, g(3)=3$ and $g(4)=4$. Therefore, $g=\operatorname{Id}_{N_{6}}$. This proves the claim.

Now, let $f \in \operatorname{Aut}\left(N_{6}\right)$. Then, from the action of $f$ on $\mathcal{E}_{3,6}, f(1)=1,2,3$ or 4 .

If $f(1)=1$ then, by Claim $2, f=\operatorname{Id}_{N_{6}}$. If $f(1)=2$ then $\left(\alpha_{1} \circ f\right)(1)=1$ and hence, by Claim 2, $\alpha_{1} \circ f=\operatorname{Id}_{N_{d}}$. This implies that $f=\alpha_{1}^{-1}=\alpha_{1}$. Similarly, $f(1)=3$ implies $f=\alpha_{2}$ and $f(1)=4$ implies $f=\alpha_{1} \circ \alpha_{2}$. Thus, $\operatorname{Aut}\left(N_{6}\right)=\left\langle\alpha_{1}, \alpha_{2}\right\rangle \cong \mathbb{Z}_{2} \times \mathbb{Z}_{2}$.

For an orientable closed surface $S, \mathrm{Hom}^{+}(S)$ denotes the group of orientation-preserving homeomorphisms of $S$. We say that a finite group $G$ acts (effectively and orientably) on $S$ if 
there is a monomorphism $\varepsilon: G \rightarrow \operatorname{Hom}^{+}(S)$. For an orientable combinatorial 2-manifold $M$, if $\sigma \in \operatorname{Aut}(M)$ then $\sigma$ induces an homeomorphism $|\sigma|:|M| \rightarrow|M|$ given by $|\sigma|(f):=f \circ \sigma$. Let $\iota: H \rightarrow \operatorname{Aut}(M)$ be a monomorphism. If $|\iota(\alpha)| \in \operatorname{Hom}^{+}(|M|)$ for each $\alpha \in H$ then, we have an action $\varepsilon: H \rightarrow \operatorname{Hom}^{+}(|M|)$, namely, $\varepsilon(\alpha):=|\iota(\alpha)|$. In this case, we say that $H$ acts simplicially on $|M|$. In [6], Broughton classified all the finite groups which act on the double-torus. Among others, $\mathbb{Z}_{2} \times \mathbb{Z}_{2}, \mathbb{Z}_{6}, D_{3}$ and $D_{6}$ act on the double-torus. Using Lemma 2.2, we get actions of $\mathbb{Z}_{2} \times \mathbb{Z}_{2}, \mathbb{Z}_{6}, D_{3}$ and $D_{6}$ on the double-torus. More explicitly, we have the following.

LEMma 2.3. $\mathbb{Z}_{6}$ acts simplicially on $\left|N_{1}\right|$ and on $\left|N_{2}\right|, D_{6}$ acts simplicially on $\left|N_{3}\right|, D_{3}$ acts simplicially on $\left|N_{5}\right|$, and $\mathbb{Z}_{2} \times \mathbb{Z}_{2}$ acts simplicially on $\left|N_{6}\right|$.

Proof. Since $N_{i}$ is orientable, $N_{i}$ has a coherent orientation for $1 \leq i \leq 6$. Fix a coherent orientation on $N_{i}$ (say, the faces in the counterclockwise direction in the pictures as the positively oriented faces, namely, $+\{0,1,2\}=\langle 012\rangle$ in $N_{1}$ ) (see [7] for definitions and notations).

If $\sigma=(0,1, \ldots, 11)$, then $\sigma \in \operatorname{Aut}\left(N_{i}\right)$ and $|\sigma|:\left|N_{i}\right| \rightarrow\left|N_{i}\right|$ is orientation reversing for $i=1,2$. But, $\left|\sigma^{2}\right|:\left|N_{i}\right| \rightarrow\left|N_{i}\right|$ is orientation preserving. So, $\left|\sigma^{2}\right| \in \operatorname{Hom}^{+}\left(\left|N_{i}\right|\right)$ for $i=1,2$. Since the order of $\sigma^{2}$ is $6, \overline{1} \mapsto \sigma^{2}$ defines a monomorphism $\iota: \mathbb{Z}_{6} \rightarrow \operatorname{Aut}\left(N_{i}\right)$ for $i=1,2$. This proves the first statement.

If $\tau=(1,4)(2,3)(8,9)(6,11)(0,5)(7,10)$ then $\operatorname{Aut}\left(N_{3}\right)=\left\langle\sigma^{2}, \tau\right\rangle$. Easy to check that $\left|\sigma^{2}\right|$ and $|\tau|$ are in $\operatorname{Hom}^{+}\left(\left|N_{3}\right|\right)$. The second statement follows from the fact that $\left\langle\sigma^{2}, \tau\right\rangle \cong D_{6}$.

If $\alpha, \beta, \alpha_{1}$ and $\alpha_{2}$ are as in Lemma 2.2 then, $|\alpha|,|\beta| \in \operatorname{Hom}^{+}\left(\left|N_{5}\right|\right)$ and $\left|\alpha_{1}\right|,\left|\alpha_{2}\right| \in$ $\operatorname{Hom}^{+}\left(\left|N_{6}\right|\right)$. Since, by Lemma 2.2, $\operatorname{Aut}\left(N_{5}\right)=\langle\alpha, \beta\rangle \cong D_{3}$ and $\operatorname{Aut}\left(N_{6}\right)=\left\langle\alpha_{1}, \alpha_{2}\right\rangle \cong$ $\mathbb{Z}_{2} \times \mathbb{Z}_{2}$, the last two statements follow.

\section{Proof of the theorem}

LEMMA 3.1. Let $M$ be a combinatorial 2-manifold on 12 vertices. Let $x, y$ and $z$ be three distinct vertices in $M$. If the degree of each vertex in $M$ is 7 then the number of faces in $\operatorname{st}(x) \cup \operatorname{st}(y)$ is $\geq 12$ and the number of faces in $\operatorname{st}(x) \cup \operatorname{st}(y) \cup \operatorname{st}(z)$ is $\geq 15$.

Proof . Let $m$ be the number of faces in st $(x) \cup \operatorname{st}(y)$. If $x y$ is an edge then $m=2+5+5=$ 12. If $x y$ is not an edge then $m=7+7=14$.

Let $n$ be the number of faces in $\operatorname{st}(x) \cup \operatorname{st}(y) \cup \operatorname{st}(z)$. If $x y z$ is a face then $n=1+3+$ $(3 \times 4)=16$. If $x y z$ is not a face but $x y, x z$ and $y z$ are edges then $n=3 \times 2+3 \times 3=15$. If one of $x y, x z$ and $y z$ is a non-edge, say $x y$ is a non-edge then the number of faces in $\operatorname{st}(x) \cup \operatorname{st}(y)$ is 14 and hence $n \geq 17$.

Proof of Theorem 1. Parts (a) and (b) follow from Lemmas 2.1 and 2.2.

Part (c) : Let $M$ has $n$ vertices and the degree of each vertex is $d$. Then the number of edges is $n d / 2$ and hence the number of faces is $n d / 3$. Therefore $-2=\chi(M)=n-n d / 2+n d / 3=$ $n-n d / 6$. This implies that $n d$ is divisible by 6 and $6<d<n$ and hence $(n, d)=(12,7)$.

Let the vertex set of $M$ be $V=\{0,1, \ldots, 9, u, v\}$ and $\psi: V \rightarrow\{0,1, \ldots, 11\}$ be given by $\psi(i)=i$ for $0 \leq i \leq 9, \psi(u)=10, \psi(v)=11$.

Claim 1. There exist faces $a b c$ and $b c d$ such that $a d$ is a non-edge of $M$ and not all edges $a b, a c, b d, c d$ are in $G_{2}(\mathrm{EG}(M))$. 
Assume without loss of generality, that $\operatorname{lk}(0)=C_{7}(1,2, \ldots, 7)$. Then, there exists $i \in$ $\{1, \ldots, 7\}$ such that $\{i, i+1, j\}$ is not a face in $M$ for all $j \in\{1, \ldots, 7\}$, where the addition is modulo 7. (Otherwise, $\{i, i+1, i+3\},\{i, i+1, i+4\}$ or $\{i, i+1, i+5\}$ is a face for each $i \in\{1, \ldots, 7\}$, then we get 14 faces through the vertices $0,1, \ldots, 7$. Hence the number of faces through the remaining four vertices is 14. This is not possible by Lemma 3.1). So, we can assume without loss of generality that 128 is a face.

If one of $01,02,18,28$ is not in $G_{2}(\mathrm{EG}(M))$ then 012 and 128 are as required. So, assume that 01, 02, 18, 28 are in $G_{2}(\operatorname{EG}(M))$. Since 2 and 7 are in the links of 0 and 1 , we may assume that $\operatorname{lk}(1)=C_{7}(7,0,2,8,9, u, v)$. Similarly, since 1 and 3 are in the links of 0 and 2 , the link of 2 must be of the form $C_{7}(8,1,0,3, p, q, r)$, where $\{p, q, r\}=\{9, u, v\}$. Then 12 is in $G_{5}(\operatorname{EG}(M))$ and 27 is a non-edge of $M$. These arguments imply that 012 and 017 are as required. This proves the claim.

By Claim 1, we may assume that 012, 128 are faces, where 08 is a non-edge of $M$ and 01 is not in $G_{2}(\mathrm{EG}(M))$. Also, assume that $\operatorname{lk}(0)=C_{7}(1,2, \ldots, 7)$. Then, $\operatorname{lk}(1)=$ $C_{7}\left(8,2,0,7, u_{1}, u_{2}, u_{3}\right)$ for some $u_{1}, u_{2}, u_{3} \in V$ and $\left\{u_{1}, u_{2}, u_{3}\right\} \neq\{9, u, v\}$. Since $M$ is orientable, $\left(u_{1}, u_{2}\right),\left(u_{2}, u_{3}\right) \notin\{(3,4),(4,5),(5,6)\}$.
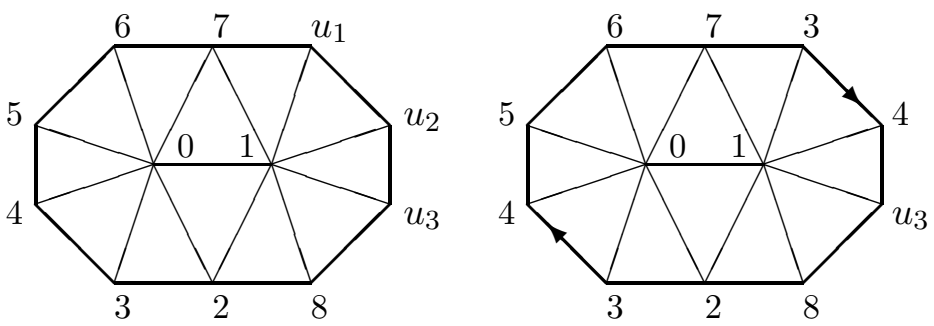

Now, it is easy to see that (up to an isomorphism) $\left(u_{1}, u_{2}, u_{3}\right) \in\{(3,6,9),(3,9,5)$, $(3,9,6),(3,9, u),(4,3,9),(4,6,5),(4,9,6),(4,9, u),(5,4,9),(5,9, u),(9,4, u),(9,5,4)$, $(9,5, u)\} \bigcup\{(3,5,4),(3,5,9),(3,6,4),(3,6,5),(4,3,5),(4,3,6),(4,6,3),(4,6,9),(4,9,5)$, $(5,3,6),(5,3,9),(5,9,3),(5,9,4),(9,3,6),(9,6,3)\} \bigcup\{(3,9,4),(4,9,3),(5,4,6),(5,9,6)$, $(9,3,5),(9,3, u),(9,4,3),(9,4,6),(9,5,3),(9,6,4),(9,6,5),(9,6, u),(9, u, 3),(9, u, 4)$, $(9, u, 5),(9, u, 6)\}=A \cup B \cup C$, say.

Claim 2. A combinatorial 2-manifold corresponding to any $\left(u_{1}, u_{2}, u_{3}\right) \in C$ is isomorphic to a combinatorial 2-manifold corresponding to some $\left(u_{1}, u_{2}, u_{3}\right) \in A \cup B$.
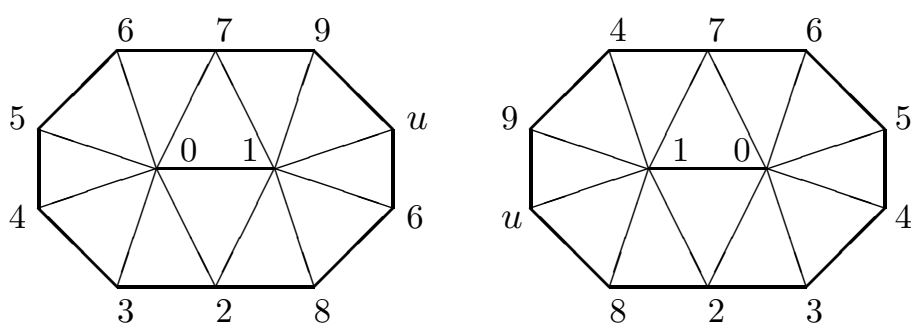

$$
(0,1)(3,8)(4, u, 5,9,6):(9, u, 6) \cong(4,9, u)
$$

Observe that the case $\left(u_{1}, u_{2}, u_{3}\right)=(9, u, 6)$ is isomorphic to the case $\left(u_{1}, u_{2}, u_{3}\right)=$ $(4,9, u)$ by the map $(0,1)(3,8)(4, u, 5,9,6)$. We denote this by $(0,1)(3,8)(4, u, 5,9,6)$ : $(9, u, 6) \cong(4,9, u)$. With this notation we have:

$(0,1)(3,8)(4, u, 5)(6,9):(9, u, 5) \cong(9,4, u), \quad(0,1)(3,8)(4, u)(5,9,6):(9,6, u) \cong(5,9, u)$, $(0,1)(2,7)(3,9)(6,8)(4,5, u):(9, u, 4) \cong(9,5, u), \quad(0,1)(3,8)(9,6,5):(9,6,4) \cong(5,9,4)$, $(0,1)(2,7)(3,5, u, 4,9)(6,8):(9, u, 3) \cong(5,9, u), \quad(0,1)(3,8)(4,9,6,5):(9,6,5) \cong(5,4,9)$, 
$(0,1)(2,7)(3,5,4,9)(6,8):(9,5,3) \cong(5,9,4)$,

$(0,1)(2,7)(6,8)(3,4,9):(9,3,5) \cong(4,9,5)$,

$(0,1)(2,7)(6,8)(5, u)(3,4,9):(9,3, u) \cong(4,9, u)$,

$(0,1)(2,7)(3,5,9,4)(6,8):(4,9,3) \cong(5,3,9)$,

$(0,1)(2,7)(6,8)(4,5,9):(3,9,4) \cong(3,5,9)$.

This proves the claim.

$$
\begin{aligned}
&(0,1)(3,8)(4,5,9,6):(9,4,6) \cong(4,9,5), \\
&(0,1)(2,7)(6,8)(3,5,9):(9,4,3) \cong(5,4,9), \\
&(0,1)(3,8)(4,5,6):(5,4,6) \cong(4,6,5), \\
&(0,1)(3,8)(4,9,5,6):(5,9,6) \cong(4,6,9),
\end{aligned}
$$

Claim 3. There is no orientable combinatorial 2-manifold corresponding to any $\left(u_{1}, u_{2}, u_{3}\right)$ in $B$.

If $\left(u_{1}, u_{2}\right)=(5,3)$ then, considering $\operatorname{lk}(5)$ and orientability of $M$, we see that, 345 or 567 is a face. This is not possible. So, $\left(u_{1}, u_{2}, u_{3}\right) \neq(5,3,6)$ or $(5,3,9)$.

If $\left(u_{1}, u_{2}, u_{3}\right)=(3,5,4)$ then, considering $\mathrm{lk}(3)$, we observe that 347 or 235 is a face. Since the set of known faces remain invariant under the map $(0,1)(2,7)(4,5)(6,8)$, we may assume that 347 is a face. Then, by using Lemma 3.1 , we get $\operatorname{lk}(3)=C_{7}(5,1,7,4,0,2,9)$, $\operatorname{lk}(4)=C_{7}(8,1,5,0,3,7, u)$ and $\operatorname{lk}(7)=C_{7}(6,0,1,3,4, u, v)$. These imply that $\operatorname{lk}(5)=$ $C_{7}(9,3,1,4,0,6, x)$, for some $x \in V$. It is easy to check that $x \neq v$. This implies $\operatorname{deg}(v)<5$. So, $\left(u_{1}, u_{2}, u_{3}\right) \neq(3,5,4)$.

If $\left(u_{1}, u_{2}, u_{3}\right)=(3,6,4)$ then $03,05,16$ and 18 are edges in $1 \mathrm{k}(4)$. Since 346, 456 and 458 can not be faces, it follows that $\operatorname{lk}(4)=C_{7}(5,0,3,8,1,6, x)$, for some $x \in V$. By using Lemma 3.1, it is easy to see that $x=9$. Then $V(\operatorname{lk}(6))=\{0,1,3,4,5,7,9\}$ and $V(\operatorname{lk}(3))=\{0,1,2,4,6,7,8\}$. These imply $0,1,3,4,6 \notin \operatorname{lk}(u) \cup \operatorname{lk}(v)$. This is not possible. Thus, $\left(u_{1}, u_{2}, u_{3}\right) \neq(3,6,4)$.

If $\left(u_{1}, u_{2}, u_{3}\right)=(4,6,3)$ then $\mathrm{lk}(1)=C_{7}(8,2,0,7,4,6,3)$. Now, considering $\mathrm{lk}(6)$ we see that 356 is a face. This implies, considering $\operatorname{lk}(3)$, that 235 a face. This is not possible, since $M$ is orientable. So, $\left(u_{1}, u_{2}, u_{3}\right) \neq(4,6,3)$.

If $\left(u_{1}, u_{2}, u_{3}\right)=(5,9,3)$ then, considering $\mathrm{lk}(5)$, we see that $459 \in M$. Now, considering $\operatorname{lk}(3)$, we see that 349 is a face. This implies $C_{4}(9,3,0,5) \subseteq \operatorname{lk}(4)$. So, $\left(u_{1}, u_{2}, u_{3}\right) \neq(5,9,3)$.

If $\left(u_{1}, u_{2}, u_{3}\right)=(5,9,4)$ then, considering $\operatorname{lk}(5)$ we see that 459 is a face. Then, $C_{3}(1,5,4)$ $\subseteq \operatorname{lk}(9)$. So, $\left(u_{1}, u_{2}, u_{3}\right) \neq(5,9,4)$.

If $\left(u_{1}, u_{2}, u_{3}\right)=(9,6,3)$ then, $\operatorname{lk}(3)=C_{7}(2,0,4,6,1,8, d)$, for some $d \in V$. Now, considering $\operatorname{lk}(6)$, we see that 467 is a face. Then, $M$ is non-orientable. So, $\left(u_{1}, u_{2}, u_{3}\right) \neq(9,6,3)$.

By similar arguments, we get $\left(u_{1}, u_{2}, u_{3}\right) \neq(3,5,9),(3,6,5),(4,3,5),(4,3,6),(4,6,9)$, $(4,9,5)$ and $(9,3,6)$. This proves Claim 3.

In view of Claims 2 and 3 , we may assume that $\left(u_{1}, u_{2}, u_{3}\right) \in\{(3,6,9),(3,9,5),(3,9,6)$, $(3,9, u),(4,3,9),(4,6,5),(4,9,6),(4,9, u),(5,4,9),(5,9, u),(9,4, u),(9,5,4),(9,5, u)\}$.

Case 1. $\left(u_{1}, u_{2}, u_{3}\right)=(3,6,9)$, i.e, $\operatorname{lk}(1)=C_{7}(8,2,0,7,3,6,9)$. Now, 05, 07, 13 and 19 are edges in $1 \mathrm{k}(6)$. Since 356,367 or 679 can not be a face, it is easy to see that $\operatorname{lk}(6)=$ $C_{7}(7,0,5,9,1,3, x)$, where $x=2,8$ or $u$.

If $x=8$ then $V(\operatorname{lk}(3))=\{0,1,2,4,6,7,8\}$. This implies that the number of faces through $\{0,1, \ldots, 9\}$ is 17 . This is not possible by Lemma 3.1 .

If $x=u$ then $02,04,16,17$ and $6 u$ are edges in $\operatorname{lk}(3)$. Since $M$ is orientable, $\operatorname{lk}(3)=$ $C_{7}(4,0,2, u, 6,1,7)$. So, $3 \notin \operatorname{lk}(v)$ and hence $7 \in \operatorname{lk}(v)$. Then $\operatorname{lk}(7)=C_{7}(4,3,1,0,6, u, v)$. This implies that $\operatorname{lk}(2)=C_{7}(u, 3,0,1,8, v, y)$, where, it is easy to see that $y=4,5$ or 9. If $y=4$ or 5 then $\operatorname{deg}(8)<7$. If $y=9$ then, considering $\operatorname{lk}(9)$ and $\operatorname{lk}(u)$, we get $C_{5}(1,2, v, u, 9) \subseteq \operatorname{lk}(8)$. So, $x \neq u$. Thus, $x=2$, i.e., $\operatorname{lk}(6)=C_{7}(7,0,5,9,1,3,2)$.

Now, by using Lemma $3.1, \operatorname{lk}(3)=C_{7}(4,0,2,6,1,7, u)$. Since $0,1,3,6 \notin \operatorname{lk}(v), 7 \in$ $\operatorname{lk}(v)$. Thus, $\operatorname{lk}(7)=C_{7}(2,6,0,1,3, u, v)$. This implies $\operatorname{lk}(2)=C_{7}(8,1,0,3,6,7, v)$. Now, 
considering the links of $u, 4$ and 5 , we see that 458 or $45 v$ is a face. Thus, $\operatorname{lk}(4)=$ $(u, 3,0,5, v, z, w)$ or $C_{7}(u, 3,0,5,8, z, w)$ for some $z, w \in V$.

Subcase 1.1. In the first case, considering $\mathrm{lk}(8)$, we get $(z, w)=(9,8)$. So, $\operatorname{lk}(4)=$ $C_{7}(u, 3,0,5, v, 9,8)$. Now, completing successively, we get $\operatorname{lk}(8)=C_{7}(2,1,9,4, u, 5, v)$ and $\operatorname{lk}(v)=C_{7}(2,7, u, 9,4,5,8)$. Then $M \cong N_{1}$ by the map $\psi \circ(1,8,7,4)(2,6, u, 3)(5, v)$.

Subcase 1.2. In the second case, it is easy to see that $(z, w)=(v, 9)$. Thus, $\operatorname{lk}(4)=$ $C_{7}(u, 3,0,5,8, v, 9)$. Now, completing successively, we get $\operatorname{lk}(8)=C_{7}(9,1,2, v, 4,5, u)$, $\operatorname{lk}(9)=C_{7}(8,1,6,5, v, 4, u)$ and $\operatorname{lk}(5)=C_{7}(9,6,0,4,8, u, v)$. Here $M \cong N_{3}$, by the map $\psi \circ(0,4,1,8,7)(2, u, v, 9,5,3)$.

Case 2. $\left(u_{1}, u_{2}, u_{3}\right)=(3,9,5)$, i.e., $\operatorname{lk}(1)=C_{7}(8,2,0,7,3,9,5)$. Now, $02,04,17$ and 19 are edges in $\operatorname{lk}(3)$. So, $\operatorname{lk}(3)=C_{7}(4,0,2,9,1,7, x)$ or $C_{7}(2,0,4,7,1,9, x)$, for some $x \in V$. The second case is isomorphic to the first case, by the map $(0,1)(2,7)(4,9)(6,8)$. So, we may assume $\operatorname{lk}(3)=C_{7}(4,0,2,9,1,7, x)$ for some $x \in V$. It is easy to see that $x=8$ or $u$.

If $x=8$ then, $\operatorname{lk}(3)=C_{7}(4,0,2,9,1,7,8)$. Now, $\operatorname{lk}(2)=C_{7}(9,3,0,1,8, y, z)$, for some $y, z \in V$. If $\{y, z\} \cap\{u, v\}=\emptyset$, then we get 18 faces outside $\operatorname{st}(u) \cup \operatorname{st}(v)$. This is not possible by Lemma 3.1. So, up to an isomorphism, $y=u$ or $z=u$. If $y=u$ then $z=v$, else the vertices $0,1,2,3,8 \notin \mathrm{lk}(v)$. So, $\operatorname{lk}(2)=C_{7}(9,3,0,1,8, u, v)$. This implies that $\operatorname{lk}(8)=C_{7}(3,4, u, 2,1,5,7)$ and $V(\operatorname{lk}(5))=\{0,1,4,6,7,8,9\}$. Then $0,1,3,5,8 \notin \operatorname{lk}(v)$. This is not possible. If $z=u$ then $y=4$ or $v$. If $y=4$ then $\operatorname{lk}(2)=C_{7}(9,3,0,1,8,4, u)$. Now, it follows that $\operatorname{lk}(8)=C_{7}(3,4,2,1,5, v, 7)$ and $\operatorname{lk}(4)=C_{7}(u, 2,8,3,0,5, v)$. This implies $\operatorname{lk}(5)=C_{7}(9,1,8, v, 4,0,6)$. Hence $\operatorname{lk}(9)=C_{7}(6,5,1,3,2, u, v)$. But then $C_{4}(v, 9,2,4) \subseteq$ $\operatorname{lk}(u)$. If $y=v$ then, $\operatorname{lk}(2)=C_{7}(9,3,0,1,8, v, u)$. It is easy to see that $\operatorname{lk}(8)=C_{7}(3,4, v, 2,1$, $5,7)$. So, $V(\operatorname{lk}(5))=\{0,1,4,6,7,8,9\}$. Then $0,1,3,5,8 \notin \operatorname{lk}(u)$. This is not possible. So, $x \neq 8$. Thus, $x=u$, i.e., $\operatorname{lk}(3)=C_{7}(4,0,2,9,1,7, u)$.

Now $\operatorname{lk}(2)=C_{7}(9,3,0,1,8, b, c)$ for some $b, c \in V$. It is easy to see that, $(b, c)=(4,6)$, $(4, u),(4, v),(6, u),(6, v),(u, 6),(u, v),(v, 4),(v, 6)$ or $(v, u)$.

If $(b, c)=(4,6)$ then $0,1,2,3,4 \notin \operatorname{lk}(v)$ and hence $\operatorname{deg}(v) \leq 6$. So, $(b, c) \neq(4,6)$. If $(b, c)=(4, v)$ then $\operatorname{lk}(4)=C_{7}(2,8, u, 3,0,5, v)$. These imply that $\operatorname{lk}(5)=C_{7}(1,8, v, 4,0,6,9)$ and $8 \in \operatorname{lk}(6)$. Then $\operatorname{lk}(8)=C_{7}(6, v, 5,1,2,4, u), \operatorname{lk}(6)=C_{7}(8, u, 9,5,0,7, v)$ and $\operatorname{lk}(9)=$ $C_{7}(u, 6,5,1,3,2, v)$. This implies $\operatorname{deg}(v)=8$. So, $(b, c) \neq(4, v)$. If $(b, c)=(v, 4)$ then $\operatorname{lk}(4)=C_{7}(2,9, u, 3,0,5, v), \operatorname{lk}(5)=C_{7}(1,9, v, 4,0,6,8)$ and $\operatorname{lk}(9)=C_{7}(u, 4,2,3,1,5, v)$. This implies that $v \in \operatorname{lk}(7)$ and hence $\operatorname{lk}(v)=C_{7}(u, 9,5,4,2,8,7)$. Then $\operatorname{lk}(7)=C_{7}(8, v, u, 3$, $1,0,6)$ and hence $C_{4}(8,5,0,7) \subseteq \operatorname{lk}(6)$. So, $(b, c) \neq(v, 4)$. By similar arguments, we see that $(b, c) \neq(4, u),(6, u),(u, 6),(v, 6)$ or $(v, u)$. Thus, $(b, c)=(6, v)$ or $(u, v)$.

Subcase 2.1. $(b, c)=(6, v)$, i.e., $\operatorname{lk}(2)=C_{7}(9,3,0,1,8,6, v)$. Then, $05,07,28$ and $2 v$ are edges in $\mathrm{lk}(6)$. Since 568 can not be a face, $\operatorname{lk}(6)=C_{7}(7,0,5, v, 2,8, d)$ or $C_{7}(v, 2,8,7,0,5, d)$, for some $d \in V$. It is easy to see that $\operatorname{lk}(6)=C_{7}(7,0,5, v, 2,8,4)$ or $C_{7}(v, 2,8,7,0,5, u)$.

If $\operatorname{lk}(5)=C_{7}(9,1,8,4,0,6, v)$ then $C_{5}(v, 5,1,3,2) \subseteq \operatorname{lk}(9)$. This is not possible. So, $\mathrm{lk}(6)=C_{7}(v, 2,8,7,0,5, u)$. Completing successively, we get $\operatorname{lk}(7)=C_{7}(6,0,1,3, u, v, 8)$, $\operatorname{lk}(u)=C_{7}(5,6, v, 7,3,4,9), \operatorname{lk}(5)=C_{7}(8,1,9, u, 6,0,4), \operatorname{lk}(8)=C_{7}(4,5,1,2,6,7, v)$ and $\operatorname{lk}(4)=C_{7}(u, 9, v, 8,5,0,3)$. Then $M \cong N_{4}$ by the map $\psi \circ(0,9,6,2,4, v, 8, u, 5)(3,7)$.

Subcase 2.2. $(b, c)=(u, v)$, i.e., $\operatorname{lk}(2)=C_{7}(9,3,0,1,8, u, v)$. Now, it is easy to see that $\operatorname{lk}(9)=C_{7}(5,1,3,2, v, y, x)$, where $(x, y)=(4,8),(4,6),(6,8)$ or $(6,4)$.

If $(x, y)=(4,8)$ then, $\operatorname{lk}(8)=C_{7}(4, u, 2,1,5, v, 9)$. This implies that $C_{6}(u, 8,9,5,0,3) \subseteq$ $\operatorname{lk}(4)$. If $(x, y)=(4,6)$ then, it is easy to see that $\operatorname{lk}(5)=C_{7}(8,1,9,4,0,6, v)$. This implies that $\operatorname{lk}(6)=C_{7}(4,9, v, 5,0,7,8)$. Then $\operatorname{deg}(8) \geq 8$. If $(x, y)=(6,8)$ then, we get $\operatorname{lk}(8)=C_{7}(6,9, v, u, 2,1,5)$. This implies $C_{4}(6,8,1,9) \subseteq \operatorname{lk}(5)$. So, $(x, y)=(6,4)$. 
Now, completing successively, we get $\operatorname{lk}(4)=C_{7}(v, 5,0,3, u, 6,9), \operatorname{lk}(5)=C_{7}(v, 4,0,6$, $9,1,8), \operatorname{lk}(u)=C_{7}(8,6,4,3,7, v, 2), \operatorname{lk}(6)=C_{7}(8, u, 4,9,5,0,7), \operatorname{lk}(7)=C_{7}(8,6,0,1,3, u, v)$, $\operatorname{lk}(8)=C_{7}(v, 7,6, u, 2,1,5)$ and $\operatorname{lk}(v)=C_{7}(7,8,5,4,9,2, u)$. Here $M \cong N_{5}$, by the map $\psi \circ(0,2)(3, v, 8,9,4)(7, u)$.

Case 3. $\left(u_{1}, u_{2}, u_{3}\right)=(3,9,6)$, i.e., $\operatorname{lk}(1)=C_{7}(8,2,0,7,3,9,6)$. Now, 02, 04, 17 and 19 are edges in $\mathrm{lk}(3)$. Since 237 and 349 can not be faces (because of orientability), $\operatorname{lk}(3)=$ $C_{7}(4,0,2,9,1,7, x)$ or $C_{7}(2,0,4,7,1,9, y)$, for some $x, y \in V$. It is not difficult to see that $\operatorname{lk}(3)=C_{7}(4,0,2,9,1,7,8), C_{7}(4,0,2,9,1,7, u), C_{7}(2,0,4,7,1,9,5)$ or $C_{7}(2,0,4,7,1,9, u)$.

If $\operatorname{lk}(3)=C_{7}(4,0,2,9,1,7,8)$ then, considering links of 8 and 6 we get $0,1,3,6,8 \notin \operatorname{lk}(v)$ and hence $\operatorname{deg}(v) \leq 6$. So, $\operatorname{lk}(3) \neq C_{7}(4,0,2,9,1,7,8)$. If $\operatorname{lk}(3)=C_{7}(2,0,4,7,1,9,5)$ then 04, 06, 23 and 39 are edges in $\operatorname{lk}(5)$. Thus 459 or 256 is a face. In both the cases, $\operatorname{deg}(v) \leq 6$. Thus, $\operatorname{lk}(3) \neq C_{7}(2,0,4,7,1,9,5)$. By similar arguments, we get $\operatorname{lk}(3) \neq C_{7}(4,0,2,9,1,7, u)$. So, $\operatorname{lk}(3)=(2,0,4,7,1,9, u)$.

Now, $\operatorname{lk}(7)=C_{7}(4,3,1,0,6, z, w)$, for some $z, w \in V$. It is easy to see that $(z, w)=(2,8)$, $(u, v),(v, 8),(9,8),(v, u),(9, v)$.

If $(z, w)=(2,8)$ then, considering $\operatorname{lk}(6)$, we get $0,1,3,6,7 \notin \operatorname{lk}(v)$. So, $(z, w) \neq(2,8)$. If $(z, w)=(u, v)$ then, considering links of $6, u, 2$ and 8 , successively, we get $C_{3}(2,8, v) \subseteq \operatorname{lk}(5)$. So, $(z, w) \neq(u, v)$. If $(z, w)=(v, 8)$ then, $0,1,7,6,8 \notin \operatorname{lk}(u)$. So, $(z, w) \neq(v, 8)$. If $(z, w)=$ $(9,8)$ then, considering the links of 6,9 and 8 we get $0,1,7,6,8 \notin \operatorname{lk}(u)$. So, $(z, w) \neq(9,8)$. Similarly, $(z, w) \neq(v, u)$. Thus, $(z, w)=(9, v)$, i.e., $\operatorname{lk}(7)=C_{7}(4,3,1,0,6,9, v)$.

Now, $\operatorname{lk}(6)=C_{7}(5,0,7,9,1,8, s)$, where, it is easy to see that $s=u$ or $v$.

Subcase 3.1. $s=u$. Then, completing successively, we get $\operatorname{lk}(9)=C_{7}(3,1,6,7, v, 5, u)$, $\operatorname{lk}(5)=C_{7}(0,4,8, v, 9, u, 6), \operatorname{lk}(8)=C_{7}(1,2,4,5, v, u, 6), \operatorname{lk}(4)=C_{7}(0,3,7, v, 2,8,5)$ and $\operatorname{lk}(2)=C_{7}(0,1,8,4, v, u, 3)$. Here, $M \cong N_{6}$ by the map $\psi \circ(0,9,3, u)(1,5, v, 7,6,4,2)$.

Subcase 3.2. $s=v$. Then, completing successively, we get $\operatorname{lk}(9)=C_{7}(1,3, u, 8, v, 7,6)$, $\operatorname{lk}(8)=C_{7}(1,2,5, u, 9, v, 6), \operatorname{lk}(v)=C_{7}(4,7,9,8,6,5, u), \operatorname{lk}(u)=C_{7}(2,3,9,8,5, v, 4)$ and $\operatorname{lk}(2)=C_{7}(0,1,8,5,4, u, 3)$. Here, $M \cong N_{2}$ by the map $\psi \circ(0,1,6,5,9, u, 3,7, v, 4,8)$.

Case 4. $\operatorname{lk}(1)=C_{7}(8,2,0,7,3,9, u)$. By similar arguments as in the previous cases, $\operatorname{lk}(3)=$ $C_{7}(4,0,2,9,1,7, v)$ and $\operatorname{lk}(2)=C_{7}(9,3,0,1,8,6,5)$ or $C_{7}(9,3,0,1,8, v, 5)$. In the first case, $M \cong N_{6}$. In the second case, $M \cong N_{4}$.

Case 5. $\operatorname{lk}(1)=C_{7}(8,2,0,7,4,3,9)$. By similar arguments as in the first three cases, $\operatorname{lk}(4)=C_{7}(7,1,3,0,5,9, u), C_{7}(7,1,3,0,5,8, u), C_{7}(7,1,3,0,5, u, 2)$ or $C_{7}(7,1,3,0,5, u, v)$.

If $\operatorname{lk}(4)=C_{7}(7,1,3,0,5,9, u)$ then $M \cong N_{6}$.

If $\operatorname{lk}(4)=C_{7}(7,1,3,0,5,8, u)$ then $\operatorname{lk}(3)=C_{7}(9,1,4,0,2, v, 6)$ or $C_{7}(9,1,4,0,2, v, u)$. In the first case, $M \cong N_{5}$. In the second case, $M \cong N_{4}$.

If $\operatorname{lk}(4)=C_{7}(7,1,3,0,5, u, 2)$ then $\operatorname{lk}(2)=C_{7}(8,1,0,3,7,4, u), \operatorname{lk}(3)=C_{7}(9,1,4,0,2,7$, $v), \operatorname{lk}(7)=C_{7}(3,2,4,1,0,6, v)$ and $\operatorname{lk}(6)=C_{7}(5,0,7, v, u, 9,8)$ or $C_{7}(5,0,7, v, 8, u, 9)$. In the first case, $M \cong N_{3}$. In the second case, $M \cong N_{1}$.

If $\operatorname{lk}(4)=C_{7}(7,1,3,0,5, u, v)$ then $\operatorname{lk}(7)=C_{7}(6,0,1,4, v, 8,2)$ or $C_{7}(6,0,1,4, v, 9,8)$. In the first case, $M \cong N_{6}$. In the second case, $M \cong N_{4}$.

Case 6. $\operatorname{lk}(1)=C_{7}(8,2,0,7,4,6,5)$. By similar arguments as in the first three cases, $\operatorname{lk}(4)=$ $C_{7}(6,1,7,5,0,3,9)$. This case is isomorphic to Case 1 by the map $(4,1,0,6,2,5,3,9,8)$.

Case 7. $\operatorname{lk}(1)=C_{7}(8,2,0,7,4,9,6)$. By similar arguments as in the first three cases, $\operatorname{lk}(4)=C_{7}(5,0,3,9,1,7, u)$ or $C_{7}(3,0,5,7,1,9, u)$. In the first case, $M \cong N_{4}$. In the second case, $M \cong N_{6}$. 
Case 8. $\operatorname{lk}(1)=C_{7}(8,2,0,7,4,9, u)$. By similar arguments as in the first three cases, $\operatorname{lk}(4)=C_{7}(3,0,5,7,1,9, v)$ or $C_{7}(5,0,3,9,1,7,8)$.

If $\operatorname{lk}(4)=C_{7}(3,0,5,7,1,9, v)$ then $\operatorname{lk}(7)=C_{7}(6,0,1,4,5, u, 8)$ or $C_{7}(6,0,1,4,5, u, v)$. In the first case, $M \cong N_{4}$. In the second case, $M \cong N_{4}$ or $N_{5}$.

If $\operatorname{lk}(4)=C_{7}(5,0,3,9,1,7,8)$ then $\operatorname{lk}(8)=C_{7}(u, 1,2,7,4,5, v)$ or $C_{7}(2,1, u, 5,4,7, v)$. In the first case, $M \cong N_{6}$. In the second case, $M \cong N_{4}$.

Case 9. $\operatorname{lk}(1)=C_{7}(8,2,0,7,5,4,9)$. By similar arguments as in the first three cases, $\operatorname{lk}(5)=C_{7}(7,1,4,0,6,8, u), C_{7}(7,1,4,0,6, u, 8)$ or $C_{7}(7,1,4,0,6, u, v)$.

If $\operatorname{lk}(5)=C_{7}(7,1,4,0,6,8, u)$ then, $\operatorname{lk}(8)=C_{7}(9,1,2, u, 5,6,3)$ or $C_{7}(u, 5,6,9,1,2, v)$. In the first case, $M \cong N_{4}$. In the second case, $M \cong N_{5}$.

If $\operatorname{lk}(5)=C_{7}(7,1,4,0,6, u, 8)$ then, $\operatorname{lk}(8)=C_{7}(9,1,2,7,5, u, 3)$ or $C_{7}(9,1,2,7,5, u, v)$. In the first case, $M \cong N_{6}$. In the second case, $M \cong N_{2}$.

If $\operatorname{lk}(5)=C_{7}(7,1,4,0,6, u, v)$ then $\operatorname{lk}(7)=C_{7}(6,0,1,5, v, 3,8)$ or $C_{7}(6,0,1,5, v, 9,8)$. In the first case, $M \cong N_{4}$. In the second case, $M \cong N_{6}$.

Case 10. $\operatorname{lk}(1)=C_{7}(8,2,0,7,5,9, u)$. By similar arguments as in the first three cases, $\operatorname{lk}(5)=C_{7}(7,1,9,4,0,6,8), C_{7}(7,1,9,4,0,6, u)$ or $C_{7}(7,1,9,4,0,6, v)$. In the first case, $M \cong N_{6}$. In the second case, $M \cong N_{2}$. In the third case, $M \cong N_{4}$ or $N_{5}$.

Case 11. $\operatorname{lk}(1)=C_{7}(8,2,0,7,9,4, u)$. Then, up to an isomorphism, $\operatorname{lk}(4)=C_{7}(u, 1,9,5,0$, $3,6)$ and $\operatorname{lk}(6)=C_{7}(5,0,7,3,4, u, v)$ or $C_{7}(7,0,5, u, 4,3,8)$. In the first case, $M \cong N_{5}$. In the second case, $M \cong N_{4}$.

Case 12. $\operatorname{lk}(1)=C_{7}(8,2,0,7,9,5,4)$. By similar arguments as in the first three cases, $\operatorname{lk}(5)=C_{7}(9,1,4,0,6,8, u)$ and $\operatorname{lk}(8)=C_{7}(2,1,4,6,5, u, v)$ or $C_{7}(4,1,2, u, 5,6, v)$. In the first case, $M \cong N_{6}$. In the second case, $M \cong N_{4}$.

Case 13. $\operatorname{lk}(1)=C_{7}(8,2,0,7,9,5, u)$. By similar arguments as in the first three cases, $\operatorname{lk}(5)=C_{7}(u, 1,9,6,0,4, v), C_{7}(9,1, u, 4,0,6,3)$ or $C_{7}(9,1, u, 4,0,6,8)$. In the first two cases, $M \cong N_{4}$. In the third case, $M \cong N_{5}$. This completes the proof.

Acknowledgement : The authors thank the anonymous referee for many useful references and comments which helped to improve the presentation of this paper. The second author thanks CSIR, New Delhi, India for its research fellowship (Award No.: 9/79(797)/2001 EMR - I).

\section{References}

[1] Altshuler, A., Bokowski, J., Schuchert, P.: Neighbourly 2-manifolds with 12 vertices, J. Comb. Th. (A) 75 (1996), 148-162.

[2] Altshuler, A., Brehm, U.: Neighbourly maps with few vertices, Discrete $\mathscr{G}$ Comput Geom. 8 (1992), 93-104.

[3] Bokowski, J., Guedes de Oliveira, A.: On the generation of oriented matroids, Discrete 83 Comput Geom. 24 (2000), 197-208.

[4] Brehm, U.; Wills, J. M.: Polyhedral manifolds, Handbook of Convex Geometry (eds. Gruber, P. M. and Wills, J. M.), Elsevier Publishers, 1993, 535-554.

[5] Bagchi, B., Datta, B.: A structure theorem for pseudomanifolds, Discr. Math. 168 (1998), $41-60$. 
[6] Broughton, S. A.: Classifying finite group actions on surfaces of low genus, J. Pure Appl. Algebra 69 (1990), 233-270.

[7] Croom, F. H.: Basic Concepts of Algebraic Topology, Springer-Verlag, New York - Heidelberg, 1978.

[8] Datta, B., Nilakantan, N.: Equivelar polyhedra with few vertices, Discrete \& Comput Geom. 26 (2001), 429-461.

[9] Datta, B., Upadhyay, A. K.: Degree-regular triangulations of torus and Klein bottle, Proc. Indian Acad. Sci. (Math. Sci.) (to appear).

[10] Edmonds, A. L., Ewing, J. H., Kulkarni, R. S.: Regular tessellations of surfaces and $(p, q, 2)-$ triangle groups, Ann. of Math. 116 (1982), 113-132.

[11] Jungerman, M., Ringel, G.: Minimal triangulations on orientable surfaces, Acta Math. 145 (1980), 121-154.

[12] Lutz, F. H.: Triangulated Manifolds with Few Vertices and Vertex-Transitive Group Actions, Thesis (TU, Berlin), Shaker Verlag, Aachen, 1999.

[13] Lutz, F. H.: Enumeration and random realization of triangulated surfaces (preprint).

[14] McMullen, P., Schulte, E.: Abstract Regular Polytopes, Cambridge Univ. Press, Cambridge, 2002.

[15] McMullen, P., Schulz, Ch., Wills, J. M.: Equivelar polyhedral manifolds in $E^{3}$, Israel J. Math. 41 (1982), 331-346.

[16] Ringel, G.: Wie man die geschlossenen nichtorientierbaren Flächen in möglichst wenig Dreiecke zerlegen kann, Math. Ann. 130 (1955), 317-326.

[17] Schulte, E., Wills, J. M.: A polyhedral realization of Felix Klein's map $\{3,7\}_{8}$ on a Riemann surface of genus 3, J. London Math. Soc. 32 (1985), 539-547.

[18] Schulte, E., Wills, J. M.: Geometric realizations for Dyck's regular map on a surface of genus 3, Discrete 83 Comput Geom. 1 (1986), 141-153.

[19] Vince, A.: Regular combinatorial maps, J. Comb. Th. (B) 35 (1983), 256-277.

Basudeb Datta and Ashish Kumar Upadhyay, Department of Mathematics, Indian Institute of Science, Bangalore 560 012, India. E-mails: \{dattab, upadhyay\}@math.iisc.ernet.in. 